\title{
The Metabolic Syndrome in Hispanics - The Role of Insulin Resistance and Inflammation
}

\author{
Pablo I. Altieri, José M. Marcial, Nelson Escobales, \\ María Crespo and Héctor L. Banchs
}

Additional information is available at the end of the chapter

http://dx.doi.org/10.5772/50337

\section{Introduction}

The acknowledgment of the metabolic syndrome (MetS) as a pathological entity is one of the most important advancements in the management of cardiovascular disease in the last 2 decades. Increasing awareness and research of this syndrome has led to a deeper understanding of how different metabolic risk factors such as inflammation, insulin resistance and vascular pathologies such as coronary heart disease (CHD) interact and aggravate one another. The existence of MetS may imply uniformity in pathology across a range of populations. However, this is not the case: the mechanisms that underlie MetS and the cardiometabolic consequences they hold may very well vary between ethnicities. The following chapter aims to encompass MetS from its most fundamental principles with a focus on inflammation and insulin resistance to the novel research pertaining to its pathophysiology and management, with an emphatic eye on the Hispanic population.

\section{Metabolic syndrome in Hispanics}

The inner workings of MetS have yet to be fully elucidated; thus it remains difficult to evaluate how they differ between specific ethnic populations. Nevertheless, it remains a possibility that the processes involved in the syndrome, such as insulin resistance and inflammation, differ in degree and function with relation to Hispanic compared to nonHispanic populations. It has been a recurring theme that the interactions between poor nutritional status, physical inactivity, and genetic predisposition might contribute to the disparities in the prevalence and characteristics of MetS and its components between ethnicities and the subgroups within; this subject has been studied to the extent that even the diagnostic criteria for MetS established by the AHA/NHLBI were challenged when 
adapted to a specific Andean population ${ }^{[1]}$. Moreover, researchers have found that a single DNA variation in the form of a guanine base pair on a gene already linked to a higher risk of Coronary Heart Disease (CHD) in other races confers a fivefold reduction in risk in AfricanAmericans ${ }^{[2]}$. Lately, research has uncovered mutations in the Brain-derived neurotrophic factor (BDNF) gene, or $B d n f$ gene, which result in human obesity ${ }^{[58]}$. Mice having a truncated long $B d n f 3^{\prime}$ UTR genetic transcript developed severe hyperphagic obesity. In these mice, the ability of the adipocytokine leptin to activate hypothalamic neurons and inhibit food intake was compromised despite normal activation of leptin receptors. All these studies, whether they involve humans or mice, provide a window into a genetic basis for MetS.

Despite the obvious limits of studying a population that does not represent the entire Hispanic world, investigations exploring cardiovascular disease and MetS in Puerto Ricans provide invaluable information in understanding the interrelationship between genetics, environment and culture in the modification of cardiovascular health. Previous data support the fact that, given the same cardiovascular risk factors, Puerto Rico has a lower prevalence of CHD than other fully industrialized countries such as the United States ${ }^{[3]}$; however, the validity of these data may not be as strong today as when published nearly 3 decades ago: recent epidemiologic data show that, although mortality from CHD and stroke has been steadily decreasing in the United States in the past 4 decades, it has been increasing in Puerto Rico ${ }^{[4]}$. On the other hand, a recent investigation that examined the medical records of 173 patients with MetS who received treatment in the Cardiovascular Center of Puerto Rico and the Caribbean showed that these patients were devoid of aggressive CHD, meaning less ventricular tachycardia, less myocardial infarctions and less strokes, and had a relatively normal lipid profile (except for a mild elevation in serum triglycerides) ${ }^{[5]}$, supporting the notion that island-based Puerto Ricans acquire a milder form of MetS than mainland populations (this notion extends to Hispanics and Caucasians living in the continental U.S.) ${ }^{[6]}$ Furthermore, several investigators have reported that the incidence of ventricular tachycardia, a complication caused by remodeling and ischemia of the heart, is lower in Puerto Rico than in the United States[7], even when adjusting for a higher prevalence of MetS in Puerto Rico ${ }^{[8]}$. Interestingly, the number of cases recorded in this study showed an increased incidence of atrial fibrillation ${ }^{[9]}$; this may be thought to be a result of differential remodelling of the left ventricle and atrial function between ethnicities. In addition, the prevalence of CHD is lower in Puerto Rico than in the United States, despite a higher incidence of Diabetes Mellitus in the island than in the U.S. $(16 \% \text { vs. } 8 \%)^{[10]}$. Nonetheless, the prevalence of CHD in Puerto Rico is increasing: In the 1980s, it was 50\% lower than in the United States; it is only $20 \%$ lower today[10]. This is most likely due to external factors such as the increasingly unhealthy diet and sedentary lifestyle of many of the island's inhabitants.

\section{Importance of the Renin-Angiotensin System (RAS)}

The RAS is a complicated and essential system in the regulation of vascular homeostasis. Angiotensin II (AngII) is cleaved from angiotensin I (AngI) by angiotensin converting 
enzyme (ACE), which is localized on the surface of endothelial cells and in the media and adventia of the aorta ${ }^{[11]}$; a soluble form of ACE is also found in plasma. AngI is formed from angiotensinogen, which is secreted from the liver and cleaved by renin, which in turn is found in the juxta-glomerular cells in the kidney ${ }^{[12]}$. The traditional RAS inhibitors, angiotensin-converting enzyme inhibitors (ACE inhibitors) and angiotensin receptor blockers (ARBs), target the main RAS axis described above. However, there are additional enzymes associated with the production of AngII, such as by Cathepsin $\mathrm{G}^{[13]}$, as well as other, more novel angiotensin molecules that serve as potential therapeutic targets: the ACE2/Ang-(1-9) axis is a new and important pathway to compensate for the vasoconstrictive and hyperproliferative RAS axis. A direct mechanism implicated in the production of these distinctive angiontensin molecules involves ACE2 ${ }^{[14]}$, a novel component of the RAS that converts AngI to Ang-(1-9) and AngII to Ang-(1-7), a peptide with vasodilator and anti-proliferative properties. The induction of ACE2 not only holds therapeutic promise by producing the anti-inflammatory Ang-(1-7), but also by reducing AngII levels, thereby conferring a twofold protection against cardiovascular remodeling from ongoing hypertension and inflammation.

Concomitant to the progression of the RAS, hyperglycemia promotes the deposition of advanced glycation end products (AGEs) that are formed from the non-enzymatic glycation of proteins and lipids after contact with reducing sugars ${ }^{[15]}$. The accumulation of AGEs is an important factor in the development and progression of vascular injury in diabetesassociated atherosclerosis. Both hyperglycemia and induction of the main RAS axis will increase oxidative stress and increase the rate of the atherosclerotic process that ultimately end in apoptosis and necrosis of myocytes ${ }^{[16,17]}$, hence propagating the deleterious effects of inflammation, insulin resistance and endothelial dysfunction.

The inhibition of the RAS by ACE inhibitors and ARBs has been mainstay therapy to reduce the onset and/or progression of hypertension, left ventricular dysfunction, diabetic renal disease and atherosclerosis. For example, inhibitors of the RAS seem to be more effective than other medications in halting the progression of dilated cardiomyopathy in hamsters that have an inherited mutation that predisposes to such a disease $\mathrm{e}^{[18]}$. In rodents, pharmacological or genetic disruption of RAS action prevents weight-gain, promotes insulin sensitivity and relieves hypertension ${ }^{[19]}$, suggesting that ACE inhibitors or ARBs may present an effective treatment for MetS in humans. In addition, when obese individuals lose weight, both adipose tissue mass and systemic RAS activity are reduced ${ }^{[2,21]}$. An increase in adipose tissue angiotensinogen has been reported in diet-induced obesity ${ }^{[22,23]}$ : further evidence that lifestyle changes are integral to targeting the underlying mechanisms of MetS.

\section{Inflammation and insulin resistance}

Systemic inflammation ${ }^{[24-25]}$ is a fundamental process in the development of cardiovascular disease in patients with MetS, and this process starts with the activation of the neurohormonal system; we have data that shows elevated intra-coronary levels of AngII and 
endothelin I (EI) in some patients with Diabetes Mellitus Type 2 (DMT2). We measured these peptides in 5 patients with DMT2 and concomitant MetS, normal coronary arteries and sub-normal ejection fraction $(49 \pm 5 \%)$, and discovered that the levels of AngII and EI were elevated in the coronary sinus (coronary efflux) and aorta of these patients when compared to the control group, which consisted of 5 patients with DMT2 but without MetS that were catheterized and found to have normal coronary arteries and a normal ejection fraction ${ }^{[26]}$. In the former, MetS group, AngII levels inside the coronary sinus and aorta were $46 \pm 18$ and $35 \pm 15 \mathrm{pg} / \mathrm{ml}$, respectively, while AngII levels were $10 \pm 2 \mathrm{pg} / \mathrm{ml}$ inside both chambers of the control group $(\mathrm{P}<0.001)$. Furthermore, in the group with MetS, the EI levels inside the coronary sinus and aorta were elevated at $14 \pm 4$ and $13 \pm 6 \mathrm{pg} / \mathrm{ml}$ in both chambers, respectively, compared to $3 \pm 1 \mathrm{pg} / \mathrm{ml}$ inside both chambers of the control group $(\mathrm{P}<0.001)$.

This elevation of AngII and EI will activate angiotensin II receptor type 1 (AT1) and produce inflammatory cytokines, increase macrophage chemo-attractants and activate reactive oxygen species that produce oxidative stress in myocytes and smooth muscle cells ${ }^{[27]}$. This will not only induce the apoptosis and necrosis of myocytes, but also promote the proliferation and migration of smooth muscle cells, resulting in the atherosclerotic lesions that increase the incidence of myocardial infarcts ${ }^{[28]}$. Likewise, AngII, acting via angiotensin II receptor type 2 (AT2), has potent pro-inflammatory, pro-oxidant and pro-thrombotic effects ${ }^{[29]}$. Moreover, it has been shown that infusion of AngII in rats increases serum levels of AGEs. The oxidative and apoptotic effects of both hyperglycemia and AngII are most likely key in inducing diabetic cardiomyopathy ${ }^{[27,28]}$, which explains why our patients with MetS have a subnormal ejection fraction, as opposed to the patients without MetS who have a normal ejection fraction, despite normal coronary arteries in both groups.

Omega-3, eicosapentaenoic acid (EPA) and docosahexaenoic acid (OHA) have also been shown to be anti-inflammatory; they are enzymatically converted to resolvins, which are very potent anti-inflammatory agents ${ }^{[30]}$. Resolvins diminish the activation and production of superoxide, increase nitric oxide and decrease inflammatory cytokines- this mechanism will counterbalance inflammation and, as a consequence, insulin resistance. It has been shown that Omega-3 in high doses will reduce the incidence of myocardial infarction and, in some patients, revert insulin resistance. We have reported the normalization of the 2-hour postprandial levels of blood sugar with the use of Omega-3[6]. The mean 2-hour post-prandial glucose levels in 10 patients decreased from a mean value of $205 \pm 40 \mathrm{mg} / \mathrm{dl}$ to $119 \pm 13 \mathrm{mg} / \mathrm{dl}$ $(\mathrm{P}<0.003)$. This change occurred after using $6000 \mathrm{mg}$ per day of pure Omega-3 for about 6 months. The effect of Omega-3 is mediated through the insulin receptors of the cells. At present, we are studying these receptors in order to explain this increase in insulin sensibility.

Adipose tissue is a hormonally active endocrine tissue, producing cytokines, which influence other body tissues. Adiponectin is one such adipocytokine that protects cardiovascular tissue from ischemic injury and increases insulin sensitivity by stimulating fatty acid oxidation, decreasing plasma triglycerides and improving glucose metabolism ${ }^{[33,34]}$. Another adipocytokine, secreted-frizzled-related-protein-5 (Sfrp5), has been found to have 
significant metabolic consequences; incorporation of Wnt signaling pathways, which classically regulate developmental processes in many organisms, in adipocytes has led to evidence that obesity induces a reduction in the Sfrp5 production along with an increase in Wnt5a expression, leading to augmented inflammatory signalling and insulin resistance ${ }^{[36]}$. Conversely, Sfrp 5 acts as an anti-inflammatory molecule, restraining the chronic inflammatory state and improving insulin sensitivity. On the other hand, tumor necrosis factor- $\alpha$ (TNF- $\alpha$ ) and interleukin-6 (IL-6) are insulin antagonizing adipocytokines ${ }^{[37]}$ that are associated to augmentation of inflammation and insulin resistance. As seen, inflammation is a complicated issue in obesity that has to be stopped due to deleterious effects produced in the cardiovascular system, such as the reduction in ejection fraction observed in our sample of patients with MetS and normal coronaries: a diabetic cardiomyopathy undoubtedly brought on by chronic inflammation and insulin resistance ${ }^{[40]}$.

Insulin resistance is a fundamental mechanism underlying MetS and its components. Insulin is an anabolic hormone that exerts its effects primarily by promoting glycogen synthesis in the liver and muscle, increasing triglyceride synthesis in adipose tissue, and augmenting protein synthesis and inhibiting proteolysis. Therefore, the consequences of insulin resistance are multiple-fold. Truly, there are other processes involved in the development of insulin resistance other than inflammation. Abnormalities in fat storage and mobilization have been implicated in the pathogenesis of insulin resistance ${ }^{[38]}$. Abdominal obesity in particular has been shown to be most associated with insulin resistance and MetS. However, it has been observed that general obesity is not universal in MetS and insulin resistance. In addition, many obese subjects do not have metabolic abnormalities. Systemic chronic inflammation [39], on the other hand, paints the most complete picture of insulin resistance as it is the result of all altered cytokine production and signaling pathways in the body. A more accessible marker for this inflammation can be obtained by measuring C-reactive protein (CRP); $40 \%$ of our patients with MetS had an elevated CRP. Clinically, each of the diagnostic component criteria of the metabolic syndrome has been associated with increased levels of CRP[41], elevation of which bears a negative prognostic implication in the population involved - this biomarker has been associated to the development of heart disease, although this observation is not totally clear ${ }^{[31,32]}$. CRP production is located in the liver, a process induced by pro-inflammatory cytokines; this non-specific marker of inflammation has an important role in the host innate defense mechanism, but also regulates the amount of inflammatory response by activating the complement system. CRP can be used to monitor the status of the inflammatory system, and has been used to monitor the effect of statins in the inflammatory process of MetS[35]. In the Jupiter trial, rosuvastatin (20mg/day) reduced the systemic marker of CRP.

Finally, a novel and important piece in the development of insulin resistance consists of aldosterone-induced insulin resistance through the increase in insulin-like growth factor-1 (IGF-1) and hybrid receptors ${ }^{[22]}$. This research has demonstrated that aldosterone induces vascular remodeling through the IGF-1 and hybrid receptors and suggests that blocking the effects of aldosterone may attenuate and reduce angiopathy in hypertensive patients with hyperinsulinemia. 


\section{Circadian rhythm and the metabolic syndrome}

Common disorders of circadian behavior and sleep, such as night-shift work and jetlag, are associated with increased hunger, decreased glucose and lipid metabolism and changes in hormonal processes involved in satiety ${ }^{[33]}$. Short-duration and poor-quality sleep have been shown to predict the development of DMT2 and obesity after age, BMI and various other confounding variables are considered and taken into account ${ }^{[44]}$. In addition, the induction of hunger may be associated to a reduction in circulating levels of leptin brought on by sleep deprivation ${ }^{[45]}$. Cardiovascular disease and hypertension are also related with sleep loss, as the risk of a fatal heart attack increases $45 \%$ in individuals who chronically sleep 5 hours per night or less ${ }^{[4]}$. Disruption of the circadian clock can lead to obesity, inflammation and insulin resistance ${ }^{[47]}$.

\section{Management}

\subsection{Diet and exercise}

Lifestyle approaches to treating and preventing MetS greatly improve metabolic parameters by reducing body weight and increasing the level of physical activity. Multiple studies of obese patients with DMT2, hypertension or hypercholesterolemia have shown that weight improves the cardiovascular profile, including glycemic control, in both diabetic and nondiabetic individuals. Furthermore, lifestyle change ${ }^{\left[{ }^{[8]}\right.}$ comprising reduced total/saturated fat intake and increased polyunsaturated fat/fiber intake have been shown to significantly reduce multiple metabolic and inflammatory parameters such as CRP, central obesity and triglyceride levels. The ATTICA ${ }^{[49]}$ epidemiological study showed that adherence to the Mediterranean diet was associated with $20 \%$ lower odds of having MetS, irrespective of age, sex, physical activity, lipids and blood pressure levels. On the other hand, consumption of a high fat diet induces changes in the fat microbia, producing inflammation that is associated with hyperphagia and an obese phenotype. In addition, data on the Hispanic and Asian diets with relation to diabetes have demonstrated that rice consumption is associated to an elevated risk of developing DMT2, presumably due to the higher glycemic index of rice when compared to whole grain ${ }^{[50]}$. Physical activity is a cornerstone in weight balance. However, only part of the beneficial effect of physical activity on the metabolic and cardiovascular profile is mediated through body weight changes. Physical activity improves insulin sensitivity, increases HDL levels, lowers blood pressure and maintains immune system health, which is very important in reducing inflammation and, as a consequence, insulin resistance ${ }^{[51]}$.

\subsection{Pharmacotherapy}

Although intensified therapeutic lifestyle modifications may prevent the onset and progression of MetS, some patients may require drug therapy. While the individual components (e.g. glucose intolerance, hypertension, dyslipidemia) are all appropriate 
targets for treatment, newer therapies that manage the syndrome centrally may benefit from such a collective approach and thus prove more effective. Although traditional approaches to the separate risk factors have proven effective, increasing attention is now being directed at the management of insulin resistance, obesity and inflammation. Orlistat, sibutramine, and rimonabant are approved for long-term treatment of obesity; however, sibutramine is known to cause secondary hypertension and thus is not the ideal choice of therapy in obese patients with MetS. As was mentioned above, statins and RAS inhibitors have proven antiinflammatory properties that may augment insulin sensitivity. Glitazones and metformin have been used increasingly over the recent years for the management of insulin resistance. The ongoing search for new strategies to combat the MetS has shed light on new molecules that may prove to be effective therapeutic targets in treating the syndrome; in vivo studies have established that atherosclerosis driven by the inhibition of stearoyl-coenzyme A desaturase 1 (SCD1), an enzyme involved in fatty acid metabolism, can be completely prevented by the omega-3 polyunsaturated fatty acids in dietary fish-oils ${ }^{[52]}$. Moreover, our data showing that in some patients, high doses of omega-3 polyunsaturated fatty acids will normalize the 2-hour post-prandial glucose levels in a sample from Puerto Rico is promising.

\subsection{Bariatric surgery}

Given that lifestyle changes and pharmacology may not be sufficient to achieve durable and effective weight loss, surgery to treat obesity and MetS has become an attractive alternative. In addition to weight loss, patients may enjoy improvement in other metabolic parameters such as insulin resistance and other obesity-related comorbidies. Bariatric surgery has been shown to reverse diabetes, hypertension, sleep apnea and hyperlipidemia ${ }^{[53]}$, however, selection of candidates must be a strict process in view of peri- and postoperative complications and costs associated with different surgical procedures.

\section{Social importance}

The economic cost of combating obesity and associated comorbidies is estimated to exceed $\$ 100$ billion annually in the United States ${ }^{[54]}$. With recent data showing the increasing burden of obesity throughout the world, it can be expected that the rates of MetS will be increasing. A study that aims to assess the independent contributions of MetS and its individual components to 10-year medical costs among a sample of elderly individuals showed that total costs to Medicare were $20 \%$ higher among participants with the MetS[55]. Another study found that risk factors for metabolic syndrome, such as obesity, high blood pressure, and elevated blood lipid levels, can increase a person's healthcare costs nearly 1.6fold, or about $\$ 2,000$ per year; for each additional risk factor those costs rise an average of $24 \%{ }^{[56]}$. These figures are even more alarming in the context of the prevalence of the MetS in relation to socio-economic position: social class has been shown to have an inverse relation to $\mathrm{MetS}^{[57]}$. 


\section{Conclusion}

In an age when millions of people are estimated to be afflicted by the MetS, new perspectives into this cluster of risk factors are imperative if we are to evolve its management: the focus on inflammation and insulin resistance is crucial in order to halt or delay the disease as soon as it is detected. Moreover, the public health of Hispanic populations throughout the world has been evidenced to pose a significant public health problem that should be addressed specifically because of the distinct metabolic characteristics this ethnicity may hold. Likewise, this approach should prompt further investigation into parallel cardiometabolic particularities in other ethnicities.

\section{Author details}

Pablo I. Altieri, José M. Marcial, Nelson Escobales, María Crespo and Héctor L. Banchs Department of Medicine and Physiology, University of Puerto Rico, Medical Sciences Campus, San Juan, Puerto Rico and The Cardiovascular Center of Puerto Rico and the Caribbean, Puerto Rico

\section{References}

[1] Medina-Lezama, J., et al. Optimal definitions for abdominal obesity and the metabolic syndrome in Andean Hispanics: the PREVENCION study. Diabetes Care 33, 1385-1388 (2010).

[2] Brian G. Kral, R.A.M., Bhoom Suktitipat, Ingo Ruczinski, Dhananjay Vaiya, Lisa R. Yanek, Arshed A. Quyyumi, Riyaz S. Patel, A Maziar Zafari, Viola Vaccarino, Elizabeth R. Hauser, William E. Kraus, Lewes C. Becker, Diane M. Becker. A common variant in the $\mathrm{CD} 2 \mathrm{KN} 2 \mathrm{~B}$ gene on chromosome $9 \mathrm{p} 21$ protects against coronary artery disease in Americans of African ancestry. Journal of Human Genetics (2011).

[3] Garcia Palmieri MR, C.R., Cruz VW, Cortes AM, Colon A, Filiberty M, Ayala AM, PAttern D, Sobrino R, Torres R, Navarro M. Risk factors and prevalence of Coronary Heart Disease in Puerto Rico. Circulation 42, 541-542 (1970).

[4] Capewell, S., et al. Cardiovascular risk factor trends and potential for reducing coronary heart disease mortality in the United States of America. Bull World Health Organ 88, 120130 (2010).

[5] Altieri P, B.H., Escobales N, Crespo M \& Figueroa Y A Less Aggressive Metabolic Syndrome In Puerto Rico than in the United States. J Investigative Med. 2009: P2.

[6] Altieri P, B.H., Escobales N \& Crespo M. . Metabolic Syndrome - Variability In Cultures and Interventional Management. 2nd World Congress On Interventional Therapies For Type 2 Diabetes. 2011: P4.

[7] Altieri, P., Garcia Palmieri MR. Sudden Death in Puerto Rico: A United States Caribbean Island. Revista Latina de Cardiologia. 1993: 14-17.

[8] Marcial J, Altieri PI, Banchs HL, Escobales N, Crespo M. Metabolic Syndrome among Puerto Ricans and others Hispanic populations. PRHSJ. 2011; 30 (3): 145-151. 
[9] Altieri P, Figueroa Y, Banchs H, Henandez Gil de Lamadrid, Escobales N, Crespo M. Higher incidence of atrial fibrillation in the metabolic syndrome: A Hispanic population study. Boletín Asociación Médica de Puerto Rico, Año 103. 2011; 4: 24-27.

[10] U.S.Government Medical Statistics-2010

[11] Armal JF, Battle T, Rasetti C, Challah M, Costerousse O, Vincaut E, Michel JB, AlheneGelas F. ACE in three tunicae of rat aorta expression in smooth muscle and effect of renovascular hypertension. AMJ Physiol 1994; 267: H1777-H1784

[12] Woodman ZL, Oppong SY, Cooks Hooper NM, Schwager SL, Brand WF, Ehlers MR, Sturrock ED. Shedding of somatic angiotensin converting enzyme (ACE) is inefficient compared with testis ACE despite cleavage at identiacal stalk sites. Biochem J. 2000; 347:711-718.

[13] Rykl, J., et al. Renal cathepsin G and angiotensin II generation. J Hypertens 24, 1797-1807 (2006).

[14] Ocaranza, M.P. \& Jalil, J.E. Protective Role of the ACE2/Ang-(1-9) Axis in Cardiovascular Remodeling. Int J Hypertens 2012, 594361 (2012).

[15] Yasunarik K, Kohono M, Kano H, Yakokawa K, Horio T, Yushikawa J. Aldose Hyperproliferation and hypertrophy of cultured rat vascular smooth muscle cell induced by high glucose. Arterioscler Thromb Vasc Biol. 1995; 15:2207-2212.

[16] Goldin, Beckman JA, Schmidt AM, Creager MA. Advanced glycation end products sparking the development of diabetic vascular injury. Circulation. 2006; 114:597-605.

[17] Guzik T, Mussa S, Gastald D, Sadowski J, Ratnatunga C, Pillai R, Channon KM. Machanism of increased vascular superoxide production in human diabetes mellitus. Circulation. 2002; 105:1656-1662.

[18] Crespo, M.J., Cruz, N., Altieri, P.I. \& Escobales, N. Enalapril and losartan are more effective than carvedilol in preventing dilated cardiomyopathy in the Syrian cardiomyopathic hamster. J Cardiovasc Pharmacol Ther 13, 199-206 (2008).

[19] de Kloet, A.D., Krause, E.G. \& Woods, S.C. The renin angiotensin system and the metabolic syndrome. Physiol Behav 100, 525-534 (2010).

[20] Strazzullo, P. \& Galletti, F. Impact of the renin-angiotensin system on lipid and carbohydrate metabolism. Curr Opin Nephrol Hypertens 13, 325-332 (2004).

[21] Engeli, S., et al. Weight loss and the renin-angiotensin-aldosterone system. Hypertension 45, 356-362 (2005).

[22] Boustany, C.M., et al. Activation of the systemic and adipose renin-angiotensin system in rats with diet-induced obesity and hypertension. Am J Physiol Regul Integr Comp Physiol 287, R943-949 (2004).

[23] Rahmouni, K., Mark, A.L., Haynes, W.G. \& Sigmund, C.D. Adipose depot-specific modulation of angiotensinogen gene expression in diet-induced obesity. Am J Physiol Endocrinol Metab 286, E891-895 (2004).

[24] Fuster V., Badimon JJ, Chesebro JH. The pathogenesis of Coronary Artery Disease and Acute Coronary Syndrome. N Engl J Med. Part 1 and 2, 1992; 326, 242-50, 310-8. 
[25] Fuster V, Lewis A. Conner Memorial Lecture. Mechanism Leading to Myocardial Infarction: In Sights from Studies of Vascular Biology. Circulation. 1994; 90:2126-46.

[26] Altieri P., Alvarado S., Banchs H., Escobales N., Crespo M. The role of Angiotensin II and Endothelin I in the cardiomyopathy of diabetic patients. J Investigative Med 2012; 81

[27] Heeneman S, Sluimer J, Daemen Mat JAP. Angiotensin converting enzyme and vascular remodeling. Circ Res. 2007; 101:441-454.

[28] Goldberg IJ, Dansky HM, Diabetic vascular disease. Arterioscler Thromb Vasc. Biol. 2006; 26:1693-1701.

[29] Kaschina E, Grzesiak A, Li J, et al. Angiotensin II Type II receptor stimulation. A novel option of therapeutic interference with the renin-angiotensin system in myocardial infarction. Circulation. 2008; 118:2523-2532.

[30] Spite M, Serham CN. Novel lipid mediators promote resolution of acute inflammationimpact of aspirin and statins. Cir Res. 2010; 107:1170-1184.

[31] Kanes R, Rosuvastatin Inflammation, C-Reactive Protein, Jupiter and Primary Prevention of Cardiovascular Disease a Perspective. Drug Des Devel Ther. 2010 Dec 9; 4: 383-413.

[32] Elkind MS, Tai Coates K, Daik MC, Saccor L. High Sensitivity C-Reactive Protein, Lipoprotein-Associated Phospholipase A 2 and Outcome After Ischemic Stroke. Arch Intern Med 2006 Oct 23; 166 (19): 2073-80.

[33] Ouchi N, Kihara S, Funahashi T, Matsuzawa Y, Walsh K. Obesity, adiponectin and vascular inflammatory disease. Curr Opin Lipidol. 2003 Dec;14(6):561-6.

[34] R Shibata et al. Adiponectin protects against myocardial ischemia-reperfusion injury through AMPK- and COX-2-dependent mechanisms. Nat Med 11. 2005; 10961103.

[35] Davignon J, Jacob RF, Mason RP. The Antioxidant Effect of Statins. Coron Artery Dis. 2004 Aug; 15(5): 251-8.

[36] Ouchi, Noriyuki; Higuchi, Akiko; Ohashi, Koji; Oshima, Yuichi; Gokce, Noyan; Shibata, Rei; Akasaki, Yuichi; Shimono, Akihiko; Walsh, Kenneth. Sfrp5 Is an AntiInflammatory Adipokine That Modulates Metabolic Dysfunction in Obesity. Science 2010; 329: 454-457.

[37] Pittas, A.G., Joseph, N.A. \& Greenberg, A.S. Adipocytokines and insulin resistance. J Clin Endocrinol Metab 89, 447-452 (2004)..

[38] Lewis GF, Carpentier A, Adeli K, Giacca A. Disordered fat storage and mobilizatin in the pathogenesis of insulin resistance and Type 2 diabetes. Endocr Rev. 2002; 23:20129.

[39] Sjostrand M, Eriksson JVV. Neuroendocrine mechanisms in insulin resistance. Mol Cell Endocrinol. 2009; 297:104-111.

[40] Altieri P, Albarado S, Banchs H, Escobales N, Crespo M. The role of angiotensin II and Endothelin I in the cardiomyopathy of diabetic patients. J Investigative Med. 2012: 81. 
[41] Ridker PM, Buring JE, Cook NR \& Rifai N. C-reactive protein, the metabolic syndrome, and risk of incident cardiovascular events: an 8-year follow-up of 14,719 initially healthy American women. Circulation 2003; 107:391-397.

[42] Sherajee S, Fujita Y, Rafig K, et al. Aldosterone induces vascular insulin like growth factor I receptor and hybrid receptor. Arterioscler Thromb Vasc Biol. 2012; 32:257263.

[43] Knutson, K.L. \& Van Cauter, E. Associations between sleep loss and increased risk of obesity and diabetes. Ann N Y Acad Sci 1129, 287-304 (2008).

[44] Lumeng, J.C., et al. Shorter sleep duration is associated with increased risk for being overweight at ages 9 to 12 years. Pediatrics 120, 1020-1029 (2007).

[45] Taheri, S., Lin, L., Austin, D., Young, T. \& Mignot, E. Short sleep duration is associated with reduced leptin, elevated ghrelin, and increased body mass index. PLoS Med 1, e62 (2004).

[46] Ayas, N.T., et al. A prospective study of sleep duration and coronary heart disease in women. Arch Intern Med 163, 205-209 (2003).

[47] Challet E, Delezie J. Interactions between metabolism and circadian clocks: reciprocal disturbances. An NY Acad Sci. 2011 Dec; 1243:30-46.

[48] Bo, S., et al. Effectiveness of a lifestyle intervention on metabolic syndrome. A randomized controlled trial. J Gen Intern Med 22, 1695-1703 (2007).

[49] Panagiotakos, D.B., et al. Impact of lifestyle habits on the prevalence of the metabolic syndrome among Greek adults from the ATTICA study. Am Heart J 147, 106-112 (2004).

[50] Sun A, Spegelman D, Van Dam R et. al. White rice, brown rice, and risk of diabetes type 2 in U.S. men and women. Arch Inter Med. 2010; 170:961-9.

[51] Walsh NP, Gleeson M, Pyne DB et. al. Position statement. Part two: Maintaining immune health. Exerc Immunol Rev. 2011; 17:64-103.

[52] Brown, J.M., et al. Combined therapy of dietary fish oil and stearoyl-CoA desaturase 1 inhibition prevents the metabolic syndrome and atherosclerosis. Arterioscler Thromb Vasc Biol 30, 24-30 (2010).

[53] Buchwald, H., et al. Bariatric surgery: a systematic review and meta-analysis. JAMA 292, 1724-1737 (2004).

[54] Wolf, A.M. \& Colditz, G.A. Current estimates of the economic cost of obesity in the United States. Obes Res 6, 97-106 (1998).

[55] Curtis, L.H., et al. Costs of the metabolic syndrome in elderly individuals: findings from the Cardiovascular Health Study. Diabetes Care 30, 2553-2558 (2007).

[56] Boudreau, D.M., et al. Health care utilization and costs by metabolic syndrome risk factors. Metab Syndr Relat Disord 7, 305-314 (2009).

[57] Ramsay, S.E., Whincup, P.H., Morris, R., Lennon, L. \& Wannamethee, S.G. Is socioeconomic position related to the prevalence of metabolic syndrome?: influence of social class across the life course in a population-based study of older men. Diabetes Care 31, 2380-2382 (2008). 
[58] Liao, G.Y., et al. Dendritically targeted Bdnf mRNA is essential for energy balance and response to leptin. Nat Med 18, 564-571 (2012). 